\title{
LOS GRUPOS DE RENOVACIÓN DE LA ENSEÑANZA DE LAS MATEMÁTICAS EN ESPAÑA
}

\author{
RENOVATION GROUPS OF MATHEMATICS TEACHING IN SPAIN
}

\author{
Maria Teresa González Astudillo ${ }^{1, *}$
}

\begin{abstract}
RESUMEN: Los grupos de renovación de enseñanza de las matemáticas surgen en España a mediados de los años 70 del siglo XX. Inicialmente, eran grupos muy locales que, a través de las relaciones que establecieron entre ellos y con educadores de otros países mostraron una forma diferente de enseñar las matemáticas, en la que se nota la influencia de las corrientes internacionales. En este artículo se hace un repaso de los grupos más reconocidos y se muestran sus ideas acerca de la enseñanza-aprendizaje de las matemáticas a través de una de sus publicaciones.
\end{abstract}

Palabras-clave: Matemáticas. Grupos de renovación. Enseñanza. Aprendizaje.

ABSTRACT: The renovation groups of mathematics teaching emerged in Spain in the mid70 s of the 20th century. Initially, they were very local groups that, through the relationships they established among themselves and with educators from other countries, showed a different way of teaching mathematics, in which the influence of international currents is noted. This article reviews the most recognized groups and shows their ideas about the teaching-learning of mathematics through one of their publications.

Keywords: Mathematics. Renovation groups. Teaching. Learning.

Trabajo financiado por la Consejería de Educación, como apoyo a los GIR de las Universidades Públicas de Castilla y León, a iniciar en 2019 bajo el proyecto SA050G19.

1.Universidad de Salamanca - Departamento de Didáctica de la Matemática y Didáctica de las Ciencias Experimentales - Salamanca, España.

^Autora correspondente: maite@usal.es

Número temático organizado por Wagner Rodrigues Valente 


\section{Introducción}

En este artículo se pretende rescatar la contribución de los grupos de renovación de la enseñanza de las matemáticas en España en un momento que impulsó un cambio sustancial en cuanto a la implicación de los profesores tanto en le renovación de los métodos, recursos e incluso formación en relación con los procesos de enseñanza-aprendizaje.

Estos grupos surgieron en España en un momento especialmente interesante de cambio en todos los niveles: político, social, económico y educativo. Entre los hitos que marcaron este momento hay que recordar que en el año 1978 se promulga la actual Constitución Española que supone la instauración de la democracia frente a la dictadura que había imperado hasta el momento.

Otro elemento que influyó en gran medida en el impulso de este movimiento fue la normalización de las relaciones con instituciones extranjeras y el acceso a documentos y publicaciones internacionales. Además de otros estudios, uno de los documentos clave en todo este movimiento fue la publicación en el año 1980 del Informe Cockcroft.

Estos movimientos surgieron a finales de los años 70 y tuvieron su mayor auge en los años 80 . Inicialmente, surgieron con un grupo de profesores más bien pequeño que se reunía periódicamente y elaboraron materiales para el aula que experimentaron y publicaron para dar a conocer sus experiencias a otros profesores.

Aunque se crearon numerosos grupos, entre ellos hay que destacar, por su trayectoria e impacto, al Grupo Cero, de Valencia, al Grupo Zero, de Barcelona, al Grupo Azarquiel, de Madrid, los grupos andaluces Thales y Epsilon (GONZÁLEZ, 2018), el Grupo Newton, de Canarias, o el Grupo Perímetre.

Posteriormente, estos grupos se fueron reconvirtiendo en sociedades de profesores de matemáticas que aglutinaron a los profesores de matemáticas tanto de primaria como secundaria de una región, comunidad autónoma o ciudad. Estas sociedades se encuentran activas actualmente y realizan muchas actividades como reuniones, congresos, publicación de revistas, celebración del día de las matemáticas, olimpiadas matemáticas etc.

En este documento nos vamos a centrar en los inicios de estos grupos que se implicaron proyectos locales.

\footnotetext{
Este es el caso, por ejemplo, del Proyecto "Granada-Mats" en la década de los 70 para las Matemáticas de la EGB, o el Proyecto "De 12 a 16" del año 84 para las Matemáticas en Secundaria. También caben bajo este apartado los proyectos elaborados por el Colectivo Rosa Sensat o las propuestas realizadas por el Movimiento de Escuela Popular. Todos estos proyectos se caracterizan por haber implicado a un grupo de escuelas o centros que han actuado coordinadamente en una región y que han compartido un esquema de trabajo. En estos casos, se ha fomentado la creación y empleo de material propio (RICO, 1990, p. 30).
}

Se han utilizado fundamentalmente fuentes secundarias en forma de artículos que se citan en el texto y que aparecen en las referencias y como fuentes primarias se ha recurrido al libro publicado por el Grupo Cero de Valencia publicado en el año 1984 y que lleva por título "De 12 a 16. Un proyecto de curriculum de matemáticas". Para analizar este libro se ha realizado un análisis de contenido. Primero se ofrece una visión general del libro indicando los apartados en los que está organizado y después se establecieron diferentes categorías que resultan determinantes para caracterizarlo: influencias internacionales recibidas, el material didáctico, el papel de los algoritmos, la resolución de problemas y entre los contenidos matemáticos se hace una mención especial a los de Geometría. 
Para profundizar en las premisas de las que partían estos grupos y su filosofía analizaremos el documento correspondiente a "De 12 a 16. Un proyecto de curriculum de matemáticas" que publicó el Grupo Cero de Valencia en el año 1984. Este es un documento sumamente ilustrativo ya que se dan numerosas indicaciones de la puesta en práctica de las ideas que surgieron en el seno del grupo y que posteriormente se han ido asumiendo por parte de los profesores de matemáticas.

\section{Los Grupos de Renovación Pedagógica de Matemáticas en España}

A mediados de los años 70 del siglo XX, profesores de diferentes lugares de España, preocupados por la educación matemática, sobre todo en la educación secundaria pero también en la primaria, empezaron a formar grupos de profesores, con una idea bien clara de que había que modificar la enseñanza de las matemáticas; comenzaron movimientos de renovación de la enseñanza de las matemáticas. En esa época, la enseñanza de las matemáticas estaba determinada por el movimiento de la matemática moderna. El grado de abstracción, el énfasis en las estructuras de las matemáticas, los ejercicios repetitivos y descontextualizados, no favorecían el interés hacia las matemáticas y se impuso el fracaso hacia las matemáticas.

Todo este movimiento se origina con el nacimiento del grupo Cero de Valencia en el año 1975. En aquel momento, seis profesores publicaron un artículo titulado ¿para qué las matemáticas? en el que se planteaba la situación de las matemáticas y su enseñanza en España y se daban algunas pautas de ideas ciertamente novedosas. Quizá uno de los aspectos más característicos fue el uso de la heurística que ya había desarrollado ampliamente el profesor Pedro Puig Adam. ${ }^{1}$

El grupo Zero de Barcelona surge a raíz de una escuela de verano celebrada en el año 1975 en la que un grupo de profesores valencianos impartió un curso que llevaba por título ¿Por qué las matemáticas? con claras influencias de las ideas de Hans Freudenthal ${ }^{2}$ (AZCÁRATE; CASTELLÓ; LLADÓ, 2010). Este momento fue de gran interés, pues unos meses después fallecería Franco y, además, de una transición política hacia una democracia parlamentaria, el ansia de libertad, de justicia y de una educación para todos que alcanzara a los jóvenes de las clases trabajadoras, impulsó a estos profesores a implantar experiencias innovadoras para mejorar la enseñanza y el aprendizaje de las matemáticas. Sus ideas estaban dirigidas hacia dos aspectos: por un lado, en los contenidos criticando el método deductivo y la excesiva formalidad de la matemática moderna y, por otro, la gestión del aula buscando formas de interacción entre el profesorado, los estudiantes y la materia. Con ello, evidentemente lo que se pretendía era hacer las matemáticas más cercanas a los estudiantes y que fuera una actividad humana y no el resultado de la historia de las matemáticas.

A raíz de una pequeña reunión previa, se gestó en el año 1978 la sociedad canaria de profesores de matemáticas, que recibió el nombre de Sociedad Isaac Newton. En sus fines fundacionales estaba la realización de actividades de formación y de investigación orientadas a la formación y actualización delos profesores de matemáticas, el intercambio de experiencias entre profesores, la organización de conferencias, seminarios, cursos y publicaciones para lograr los fines, incluso la defensa de los intereses profesionales de los profesores (BALBUENA, 2010). Esto cristalizó en la creación de un boletín con noticias y artículos de interés, y la celebración de jornadas periódicas para el intercambio de experiencias y la discusión de la situación de la enseñanza y el aprendizaje de las matemáticas. El boletín se transformó en el año 1981 en una revista denominada Números que continúa publicándose en la actualidad y que, este año, ha llegado a su número 105. La creación de esta sociedad impulsó a otros grupos a crear sociedades en diversos lugares de España y juntos empezaron la celebración de las JAEM (Jornadas para el Aprendizaje y la Enseñanza de las Matemáticas). Dada la cantidad de sociedades que se crearon, finalmente se unieron en una federación española de sociedades de profesores de matemáticas (FESPM) en el año 1989. 
Los orígenes de la sociedad Thales de profesores andaluces de matemáticas también están en las escuelas de verano celebradas en Barcelona. En esta comunidad había diferentes grupos de profesores en diferentes ciudades. Así en Granada estaba el equipo de investigación Granada-Mats liderado por el profesor Luís Rico que propuso una enseñanza innovadora de las matemáticas amparándose en la Ley General de Educación de 1970. Miembros de dicho grupo, crearon en el año 1984 la Asociación de Profesores de Matemáticas de Andalucía (APMA). Dicha asociación publicaba periódicamente una revista de enseñanza de las matemáticas llamada Epsilon. Por otro lado, a raíz de la celebración de una escuela de verano en la que participaron el Grupo Zero y la Sociedad Isaac Newton se creó el Colectivo de Didáctica de las Matemáticas de Sevilla. Dicho colectivo impulsó la creación de la Sociedad Andaluza de Profesores de Matemáticas que finalmente vio la luz en el año 1981. Ambas sociedades se fusionaron en el año 1987 creando la Sociedad Andaluza de Educación Matemática Thales (SAEM) y Epsilon sería la revista de la sociedad (PÉREZ, 2010).

Un poco más tardío fue el nacimiento del Grupo Azarquiel de Matemáticas de Madrid (GAMMA) cuyos orígenes están en algunas reuniones de profesores de secundaria en el curso 1980-1981. La influencia de los Grupos Cero y Zero fue decisiva en la constitución de este grupo que les llevó, al igual que a los demás, a elaborar sus propios materiales que experimentaron, intentando hacer clases activas, con la realización de actividades en contexto que motivaran a los estudiantes (ALONSO, 2010). Inicialmente sus temas de investigación fueron el uso de la calculadora y el impulso de la estadística.

Un caso un poco diferente fue el del grupo Perímetre. Inicialmente este grupo se formó con maestros de educación primaria y posteriormente incorporó a otros de educación infantil y secundaria. Al igual que otros grupos, su interés radicaba en el hecho de compartir experiencias que culminarían finalmente en la elaboración de materiales para dar soluciones a los problemas de la enseñanza de las matemáticas (CANALS; RUBIROLA; FERNÁNDEZ, 2010). También organizaron jornadas para difundir sus materiales y fomentar la innovación en el aula.

Estos grupos elaboraron materiales docentes (libros, documentos, material didáctico) para acercar las matemáticas a los alumnos. Inicialmente, comenzaron esta labor realizando lecturas y discusiones sistemáticas de materiales de otros matemáticos preocupados por la enseñanza de las matemáticas como fueron Puig Adam, Félix Klein, ${ }^{3}$ Morris Kline ${ }^{4}$ o Andréi Kolmogorov, ${ }^{5}$ pero también recurrieron a los libros de School Mathematical Project, los libros de Emma Castelnuovo ${ }^{6}$ o los materiales de Claude Janvier. ${ }^{7}$

Los grupos eran dinámicos, se reunían periódicamente, se elaboraban materiales que se llevaban a las aulas, se analizaba su implementación y resultados y se reelaboraban hasta que se consideraba que materiales adecuados para su uso en el aula. Además, se establecieron relaciones entre los diferentes grupos y empezaron a organizar reuniones como las JAEM ya mencionadas y cuya primera edición se celebró en Barcelona en el año 1981. Estas jornadas aún continúan celebrándose (la próxima está prevista para el año 2021 en Valencia). Estos grupos participaron en congresos internacionales como la CIAEM, el ICME o las jornadas hispano lusas lo que les permitió estar en contacto con profesores e investigadores de otros países que tuvieron una gran influencia en las ideas y las producciones de estos grupos como los ya mencionados, u otros como: Luís Santaló, ${ }^{8}$ Claude Gaulin, ${ }^{9}$ Freudenthal o Mialaret. ${ }^{10}$

Ideas como matemáticas significativas, interdisciplinariedad, resolución de problemas reales, trabajo cooperativo, creatividad, modelos matemáticos, el uso de la calculadora o la historia de la matemática son aspectos importantes en la generación de los materiales didácticos que se elaboraron en aquel momento.

En los años 90, la reforma de la enseñanza secundaria realizada en España incorporó muchas de las ideas gestadas durante esta época. 


\section{El Proyecto de 12 a 16}

Este proyecto se desarrolló durante tres años (1985-1988) y en él colaboraron treinta profesores de diez centros públicos de primaria y diecinueve de secundaria. En total alcanzaron a trabajar con 700 alumnos de $\mathrm{EGB}^{11}$ y 900 de BUP ${ }^{12}$ de Valencia. Finalmente, se publicaron los textos en los que se proponen numerosas actividades para alumnos y profesores. Las ideas principales sobre la enseñanza de las matemáticas que se reflejan en esos materiales son:

- Desplazar el centro de gravedad de la enseñanza de la materia al alumno;

- Considerar al profesor como un activador de conocimientos;

- Considerar el aprendizaje a partir del conflicto cognitivo;

- Organizar los contenidos matemáticos en redes conceptuales;

- Centrar la actividad en la resolución de problemas;

- Utilizar materiales manipulativos para el descubrimiento;

- Considerar la evaluación para diagnosticar problemas.

Uno de los libros que se publicaron y que recoge las ideas fundamentales en las que se basó todo el proyecto fue el titulado "De 12 a 16. Un proyecto de curriculum de matemáticas" del año 1984. En la publicación de dicho libro participaron los profesores: Eliseo Borrás, $M^{\mathrm{a}}$ Elisa Carrillo, José Luís Domingo, Francisco Hernán, Vicente Calixto, Magda Morata, Juan Carlos Orero, Luís Puig, Ángel Salar y el proyecto fue financiado por la Subdirección General de Perfeccionamiento del Profesorado del Ministerio de Educación de España. Este es el libro sobre el que realizaremos el análisis para entender cuáles eran los fundamentos en los que basaba el Grupo Cero su idea de los procesos de enseñanza y aprendizaje de las matemáticas.

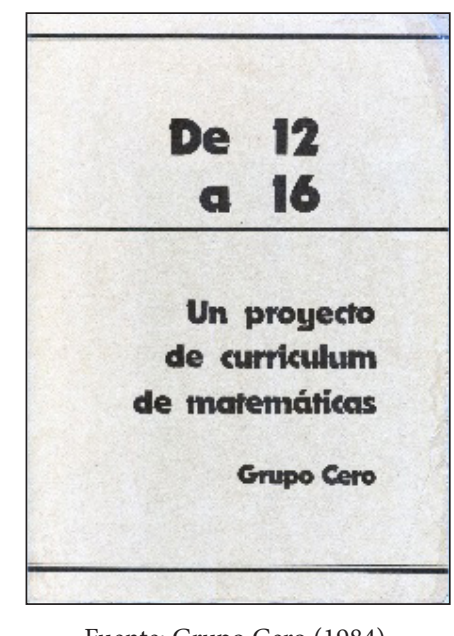

Fuente: Grupo Cero (1984).

Figura 1. Portada del libro.

El libro está organizado en nueve capítulos que se completan con un listado de bibliografía y un índice general.

Se comienza estableciendo la filosofía del proyecto para lo que incide en la noción de currículo de la que se parte, los presupuestos filosóficos en los que se basa el proyecto, un listado de las capacidades que se considera necesario desarrollar en los estudiantes, los contenidos y ciertas nociones sobre el aprendizaje y la enseñanza. 
El siguiente capítulo aborda cuestiones relativas a los métodos de enseñanza: los tipos de trabajo en el aula, las nociones relativas a hechos, algoritmos, estructuras conceptuales y estrategias generales, la idea de conflicto cognitivo, la importancia de la resolución de problemas y finaliza con algunas cuestiones relativas al aprendizaje a largo y corto plazo y el desarrollo de la memoria.

En los siguientes capítulos se abordan aspectos relativos a los contenidos de la educación secundaria. Así hay un capítulo centrado en los algoritmos y las técnicas, otro dedicado a la resolución de problemas, a las estructuras conceptuales y a la idea de investigación. Se finaliza con la propuesta del programa/plan de estudios en el que se indican los correspondientes a la aritmética, la geometría, las gráficas y tablas, el álgebra y la estadística y probabilidad. Además, se sugieren materiales para el uso en el aula.

Cada uno de los capítulos está organizado de forma que se establecen algunos aspectos teóricos que se fundamentan en lecturas de expertos en las diferentes temáticas, se plantean actividades/situaciones para realizar en el aula y, algunas de ellas, se ejemplifican mediante la resolución realizada por algunos estudiantes, comentarios sobre la actuación/gestión del profesor e indicaciones acerca del rol del docente, así como notas y sugerencias para usar las actividades propuestas en el aula.

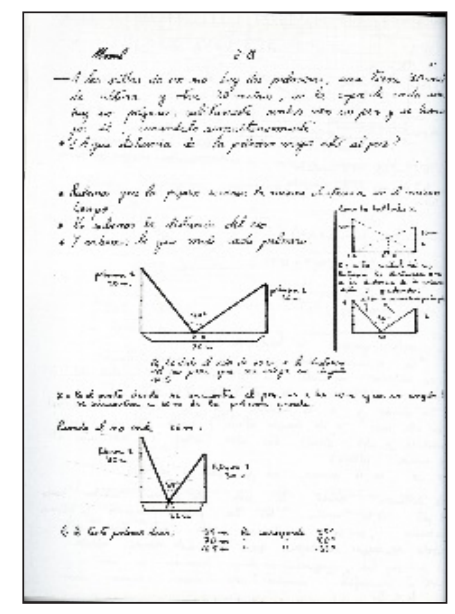

Fuente: Grupo Cero (1984).

Figura 2. Resolución de un problema de un estudiante.

Si hacemos un repaso a través de los capítulos sobre las influencias recibidas y mencionadas a lo largo del libro, así como de la bibliografía consultada, nos podemos hacer una idea de cuáles fueron las ideas principales en las que se basó esta propuesta. No mencionaremos todos pues sería demasiado extenso, pero sí algunos que nos permiten hacernos una idea global. Así encontramos a Polya ${ }^{13}$ en relación con la motivación, Bell ${ }^{14}$ para justificar el uso de diferentes tipos de actividades a realizar en el aula, Emma Castelnuovo en cuanto a la construcción de material manipulativo Polya sobre la introducción de la resolución de problemas en el aula de matemáticas, Dienes ${ }^{15}$ sobre las ideas de abstracción y generalización o Fielker ${ }^{16}$ en relación con las propuestas de enseñanza de la geometría. Además, se mencionan y usan otros proyectos o ideas acerca de la enseñanza de las matemáticas como la propuesta por el NCTM en el año 1983 o los libros del SMP (School Mathematics Project) de Cambridge.

A lo largo del libro se menciona numeroso material didáctico para el trabajo en el aula dado que se parte de la idea de "la práctica, la observación y la exploración" (GRUPO CERO, 1984, p. 360). Dicho material muchas veces se encuentra disponible en las aulas, pero también se propone su construcción por parte de los estudiantes, de forma que esta actividad forme parte del aprendizaje de las matemáticas de los estudiantes. 
Así se menciona material como: dados, barajas, ruletas, urnas y bolas de colores, cuerdas, ábacos, fichas de colores, reglas, círculos graduados, compás, tijeras, espejos, caleidoscopios, varillas de madera y alambre, mecanos, geoplanos, figuras geométricas planas, figuras de tres dimensiones, cartulinas, engranajes, balanzas, pantógrafos, plomadas, niveles, goniómetros, dominós, tramas de puntos y formas, aparato de Galton etc. Además, también se menciona y se desarrollan con cierta amplitud el uso de materiales como las calculadoras y programas informáticos.

Consideran los algoritmos como "una colección de instrucciones que, cuando se ejecutan en el orden especificado, produce el resultado correcto deseado” (GRUPO CERO, 1984, p. 27). Así, bajo el término de algoritmo se incluyen no sólo los correspondientes a la aritmética y el álgebra, sino cualquier procedimiento que se determine mediante una serie de pasos bien especificados y que concluya con el final, tanto si se refiere a cuestiones de índole aritmético-algebraica o geométrica. Cuando el algoritmo requiere pocos pasos, se refieren a él como técnica. Por ejemplo "para multiplicar por potencias de diez se corre la coma”. En relación con los algoritmos se plantean una serie de tareas (test) sobre números decimales, fracciones, lógica analizando las dificultades que plantean y las respuestas dadas por estudiantes. Dichos test se plantearon a estudiantes de $7^{\circ}$ y $8^{\circ}$ de EGB y $1^{\circ}$ de BUP a modo de investigación sobre los algoritmos. Por ejemplo, en relación con las fracciones se plantean dos preguntas (de 5) que son las siguientes (GRUPO CERO, 1984, p. 63):

2. Calcular $1 / 6$ de $3 / 4$

5. Rayar $1 / 6$ de la parte punteada del siguiente disco ¿Qué fracción del disco total se ha rayado?

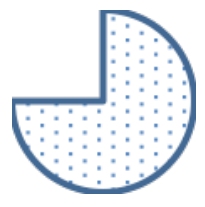

En función de la resolución que hicieron los estudiantes de las preguntas anteriores, se realiza el siguiente comentario:

\begin{abstract}
Ambas preguntas son equivalentes, pero salta a la vista que para el preguntado distan mucho de ser idénticas. Por un lado, el porcentaje de aciertos pasa de 22,5 a 32,5 en $7^{\circ}$ y de 13 a 42,5 en $8^{\circ}$ y de 27 a 47 en $1^{\circ}$. Por otro lado, el porcentaje de alumnos que no contesta pasa de 37,5 a 17,5 en $3^{\circ}$, de 31 a 16,5 en $8^{\circ}$ y de 17,5 a 2 en $1^{\circ}$. Lo que parece desprenderse de ello es que la parte de la cabeza en la que muchos alumnos albergan el buen sentido está completamente separada de la parte en que se alberga su recuerdo de cómo hacen operaciones: hay una separación entre calcular y tener sentido común (GRUPO CERO, 1984, p. 64).
\end{abstract}

Visto este comentario con la distancia que nos ha proporcionado, no sólo el paso del tiempo sino el conocimiento que hemos adquirido en didáctica de la matemática en los veinticinco años que han pasado desde entonces, nos hacen percibir los inicios de lo que luego se constituiría en la investigación en educación matemática.

En relación con los algoritmos se menciona el uso de las calculadoras y de los ordenadores, de los que se dice que se debería extraer todas las ventajas posibles. Respecto del uso de las calculadoras se indica que no sólo se deben usar como medio para comprobar resultados, sino que se menciona el potencial que tienen por tratar con el uso de datos reales, para reflexionar sobre los procedimientos de cálculo, para poner en práctica las propiedades de las operaciones o para usarlas en la resolución de problemas de forma exploratoria 
de las soluciones. En cuanto al uso de ordenadores, se hace fundamentalmente mención, tal como se indicó con anterioridad, al uso de lenguajes de programación como fue en su momento LOGO y se plantea el uso de estos programas para realizar gráficas de funciones, pero sobre todo para la enseñanza de la geometría: dibujar figuras, trabajo con vectores e incluso la simulación de juegos de azar. Dos son los procesos en los que se hace énfasis en relación con los algoritmos: la iteración y la recursión. En relación con la iteración se plantean tareas basadas en el algoritmo de Hörner, en la construcción de figuras de dos dimensiones o el juego Nim. En cuanto a la recursión se plantea la obtención de términos de la sucesión de Fibonacci, el cálculo de los coeficientes del triángulo de Pascal, el juego de las torres de Hanoi o la construcción de espirales cuadradas.

Un apartado interesante de la propuesta y que la impregna completamente es el de la resolución de problemas. La noción de problema matemático que se maneja proviene de las indicaciones del NCTM (1980) en "An agenda for action". No sólo se aborda lo que se entiende por problema, sino también las cuestiones relativas al proceso de su solución como las fases tomadas de Polya (1982). Para el Grupo Cero importa más el proceso que el resultado. Además, se tienen en cuenta algunos aspectos afectivos como el entusiasmo, la motivación o el interés, También se mencionan algunas técnicas heurísticas como: abordar un problema similar, construir tablas, empezar el problema desde atrás, explorar diferentes estrategias etc. Para cada una de ellas, se plantean problemas a modo de ejemplo de dichas técnicas, algunos de los cuáles pueden considerarse clásicos o ejemplos prototípicos. Por ejemplo, para la técnica explorar el problema desde atrás, uno de los problemas que se plantea es el relativo a la venta de huevos:

Una campesina llegó al mercado a vender huevos. La primera cliente adquirió la mitad de todos los huevos más medio huevo. La segunda la mitad de los huevos que quedaban más medio huevo. La tercera sólo compró un huevo. Con esto terminó la venta porque la campesina no tenía más huevos ¿Cuántos trajo al mercado? (GRUPO CERO, 1984, p. 113).

O para la estrategia de dividir el problema en otros subproblemas se plantea el siguiente:

Si en un triángulo equilátero de lado $10 \mathrm{~cm}$. se trazan arcos de circunferencia con centro en sus vértices y radio $5 \mathrm{~cm}$, se obtiene un triángulo equilátero curvo. Halla su área (GRUPO CERO, 1984, p. 146).

En cuanto a la enseñanza de las diferentes ramas de las matemáticas, nos centraremos en la Geometría, pues que recordar que los planes de estudio de aquel momento hacían mucho énfasis tanto en la aritmética como en el álgebra y que anteriormente la enseñanza estaba marcada por el movimiento de la matemática moderna en el que la geometría estaba relegada a un segundo plano. Las ideas plasmadas en el libro se basan en el currículo establecido por el SMP ya mencionado con anterioridad. Y en este caso la idea central es que los alumnos hagan geometría en el aula para lo que es necesario el uso de material variado, no sólo de dibujo sino también para experimentar y manipular figuras y cuerpos geométricos. En el desarrollo del programa de geometría se plantea un núcleo básico de contenidos que incluye contenido principal con actividades o sugerencias para desarrollarlo. Las actividades se plantean y se indica posibles generalizaciones y extensiones a partir de ellas. Asimismo, se abordan cuestiones relativas a las magnitudes longitud, superficie y volumen. Para hacerse una idea de la propuesta se ha seleccionado el siguiente problema de los sugeridos en el libro:

Manteniendo fijo el perímetro tenemos la posibilidad de varias el área y la forma. ¿Cuántos rectángulos diferentes puedes hacer con un perímetro de $24 \mathrm{~cm}$, habiendo de tener los lados un número entero de centímetros? (GRUPO CERO, 1984, p. 311-312). 
La idea es plantear situaciones que no sean aburridas, que los alumnos comprendan lo que hacen y que no ejecuten procedimientos de forma mecánica, que dibujen, experimenten y generalicen.

\section{Consideraciones Finales}

Los grupos de renovación pedagógica de las matemáticas fueron muy activos en España desde mediados de los años setenta y su trabajo cristalizó en la creación de sociedades y la difusión de su trabajo a través de revistas y jornadas que continúan en la actualidad.

Después de una época dominada por la matemática moderna, los profesores, fundamentalmente de secundaria, necesitaban buscar otros caminos y hacer la matemática más asequible para que pudiera llegar a más alumnos. Las relaciones entre estos grupos y con matemáticos de renombre del exterior fundamentaron esta renovación. No sólo eso. Las primeras tentativas de investigación de aquellos años fueron consolidándose $\mathrm{y}$, de hecho, muchos de aquellos profesores crearon grupos de investigación en las universidades españolas, realizaron tesis doctorales y dirigieron tesis a otros compañeros.

Aspectos como la resolución de problemas, el papel activo del alumno, el rol de facilitador del docente, la recuperación de la enseñanza de la geometría euclídea, el uso de material manipulativo, la introducción de calculadoras y ordenadores fueron aspectos que marcaron las propuestas de estos grupos de investigación.

Todas estas ideas han ido cristalizando en la enseñanza de las matemáticas en lo que puede considerarse un punto de inflexión para hacer llegar las matemáticas a más alumnos, motivando su interés y unas formas de gestión del aula más novedosas que están marcando la forma de enseñar matemáticas en la actualidad.

\section{Notas}

1. Pedro Puig Adam (1900-1960) matemático español que compaginó docencia en educación secundaria y en universidad, destacó por su interés en la didáctica de la matemática y concretamente en el uso de material manipulativo. Partícipó en la Comission Internationale pour l'edutde de L'Enseignement des Mathematiques desde 1956.

2. Hans Freudenthal (1905-1990) matemático holandés que, en 1971, octavo presidente de la International Commission on Mathematicasl Instruction (ICMI) fundó el Institute for the Development of Mathematical Education que ha destacado, entre otras cosas, por desarrollar la llamada matemática realista.

3. Félix Klein (1849-1925), matemático alemán al que se debe la definición actual de Geometría, fuel el primer presidente del ICMI y publicó en 1900 la obra "Matemáticas elementales desde un punto de vista superior" que supuso una revolución en la didáctica de la matemática.

4. Morris Kline (1908-1922), matemático estadounidense dedicado a la didáctica de la matemática. Quizá su libro más reconocido sea "El fracaso de la matemática moderna. ¿Por qué Juanito no sabe sumar?"

5. Andréi Kolmogorov (1903-1987), matemático ruso que destacó por sus aportaciones a la teoría de la probabilidad y en la estadística, siempre estuvo implicado en la educación matemática

6. Emma Castelnuovo (1913-2014), matemática italiana cuya influencia principal fue Decroly y que destacó por el uso de materiales didácticos en la enseñanza de una geometría intuitiva.

7. Claude Janvier (1940-1998), matemático canadiense muy reconocido por su aportación en relación con el uso de diferentes formas de representación en matemáticas que ha repercutido en la enseñanza de las funciones.

8. .Luís Santaló (1911-2001), matemático español exiliado en Argentina que tomó partido por la matemática moderna y se dedicó a su didáctica. 
9. Claude Gaulin (1938-2020), matemático canadiense dedicado a la formación de profesores de matemáticas con un enfoque constructivista y fomentando el uso de materiales didácticos.

10. Gaston Mialaret (1918-2016), pedagogo francés que estableció seis principios para la enseñanza de las matemáticas

11. EGB son las siglas de Enseñanza General Básica que correspondía a la enseñanza primaria en los años 1970 y 1980. Comprendía ocho cursos académicos y las edades de los alumnos iban desde los 6 a los 14 años,

12. BUP son las siglas de Bachillerato Unificado y Polivalente que correspondía con la enseñanza secundaria en España de la época. Estaba formado por tres cursos académicos y los estudiantes tenían edades comprendidas entre los 14 y los 17 años.

13. George Polya (1887-1985) fue un matemático húngaro que destacó por sus contribuciones a la enseñanza de la matemática a través de la resolución de problemas.

14. Erik Temple Bell (1883-1960) destacó sobre todo por sus escritos sobre la historia de las matemáticas.

15. Zoltan Pal Dienes (1916-2014) fue un matemático húngaro que destacó por la introducción de material manipulativo para la enseñanza de las matemáticas como los bloques lógicos.

16. David S. Fielker destaca por un gran número de libros que ha publicado sobre distintos temas de matemáticas entre los que destacan los centrados en la Geometría o en el uso de la calculadora en el aula de matemáticas.

\section{Referencias}

ALONSO, F. et al. Grupo Azarquiel: relato de una reflexión de grupo. Uno: Revista de Didáctica de las Matemáticas, Barcelona, n. 55, p. 48-56, 2010.

AZCÁRATE, C., CASTELLÓ, M. J.; LLADÓ, C. El Grupo Zero. Uno: Revista de Didáctica de las Matemáticas, Barcelona, n. 55, p. 16-26, 2010.

BALBUENA, L. La Newton. Sociedad Canaria Isaac Newton de profesores de matemáticas. Uno: Revista de Didáctica de las Matemáticas, Barcelona, n. 55, p. 27-38, 2010.

BORRÁS, E. El grupo Cero. Uno: Revista de Didáctica de las Matemáticas, Barcelona, n. 55, p. 9-15, 2010.

CANAlS, M. A.; RUBIROLA, D.; FERnÁnDEZ, X. Grupo Perímetre. Uno: Revista de Didáctica de las Matemáticas, Barcelona, n. 55, p. 57-63, 2010.

COCKCROFT, W. H. Las matemáticas sí cuentan: Informe Cockcroft. Madrid: Centro de Publicaciones/ Ministerio de Educación y Ciencia, 1985.

GONZÁLEZ, M. T. Cien números en la historia de la revista Epsilon. Epsilon, Granada, n. 100, p. 37-48, 2018.

GRUPO CERO De 12 a 16. Un proyecto de curriculum de matemáticas. Valencia: Generalitat Valenciana, 1984.

NCTM [NATIONAL COUNCIL OF TEACHERS OF MATHEMATICS]. An agenda for action: recommendations for school mathematics of the 1980s. Reston: NCTM, 1980.

PAPERT, S. Desafío a la mente. Buenos Aires: Galápago, 1982.

POLYA, B. Cómo plantear y resolver problemas. México: Trillas, 1982. 
PÉREZ, A. Con la ilusión de los años. Movimientos de renovación en Andalucía. Uno: Revista de Didáctica de las Matemáticas, Barcelona, n. 55, p. 37-47, 2010.

RICO, L. Diseño curricular en educación matemática: una perspectiva curricular. In: LLINARES, S.; SÁNCHEZ, V. (eds.). Teoría y práctica en educación matemática. Sevilla: Editorial Alfar, 1990, p. 17-62. 